\title{
Update Breast Cancer 2018 (Part 2) - Advanced Breast Cancer, Quality of Life and Prevention
}

\author{
Update Mammakarzinom 2018 (Teil 2) - Fortgeschrittenes \\ Mammakarzinom, Lebensqualität und Prävention
}

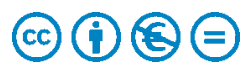

Authors

Andreas Schneeweiss ${ }^{1,2}$, Michael P. Lux ${ }^{3}$, Wolfgang Janni ${ }^{4}$, Andreas D. Hartkopf ${ }^{5}$, Naiba Nabieva ${ }^{3}$, Florin-Andrei Taran ${ }^{5}$, Friedrich Overkamp ${ }^{6}$, Hans-Christian Kolberg ${ }^{7}$, Peyman Hadji ${ }^{8}$, Hans Tesch ${ }^{9}$, Achim Wöckel ${ }^{10}$, Johannes EttI ${ }^{11}$, Diana Lüftner ${ }^{12}$, Markus Wallwiener ${ }^{2}$, Volkmar Müller ${ }^{13}$, Matthias W. Beckmann ${ }^{3}$, Erik Belleville ${ }^{14}$, Diethelm Wallwiener ${ }^{5}$, Sara Y. Brucker ${ }^{5}$, Florian Schütz ${ }^{2}$, Peter A. Fasching ${ }^{3}$, Tanja N. Fehm ${ }^{15}$

Affiliations

1 National Center for Tumor Diseases, Division Gynecologic Oncology, University Hospital Heidelberg, Heidelberg, Germany

2 Department of Obstetrics and Gynecology, University of Heidelberg, Heidelberg, Germany

3 Erlangen University Hospital, Department of Gynecology and Obstetrics, Comprehensive Cancer Center ErlangenEMN, Friedrich-Alexander University Erlangen-Nuremberg, Erlangen, Germany

4 Department of Gynecology and Obstetrics, Ulm University Hospital, Ulm, Germany

5 Department of Obstetrics and Gynecology, University of Tübingen, Tübingen, Germany

6 OncoConsult Hamburg GmbH, Hamburg, Germany

7 Department of Gynecology and Obstetrics, Marienhospital Bottrop, Bottrop, Germany

8 Department of Bone Oncology, Nordwest Hospital, Frankfurt, Germany

9 Oncology Practice at Bethanien Hospital Frankfurt, Frankfurt, Germany

10 Department of Gynecology and Obstetrics, University Hospital Würzburg, Würzburg, Germany

11 Department of Obstetrics and Gynecology, Klinikum rechts der Isar, Technical University of Munich, Munich, Germany

12 Charité University Hospital, Berlin, Campus Benjamin Franklin, Department of Hematology, Oncology and Tumour Immunology, Berlin, Germany

13 Department of Gynecology, Hamburg-Eppendorf University Medical Center, Hamburg, Germany

14 ClinSol GmbH \& Co KG, Würzburg, Germany

15 Department of Gynecology and Obstetrics, University Hospital Düsseldorf, Düsseldorf, Germany
Key words

breast cancer, treatment, metastasis, CDK4/6, PD1/PDL1, trials, risk, prevention

\section{Schlüsselwörter}

Mammakarzinom, Behandlung, Metastasen, CDK4/6, PD1/

PDL1, Studien, Risiko, Prävention

received 19.1.2018

revised 24.1.2018

accepted 24.1.2018

\section{Bibliography}

DOI https://doi.org/10.1055/s-0044-101614

Geburtsh Frauenheilk 2018; 78: 246-259 @ Georg Thieme

Verlag KG Stuttgart · New York | ISSN 0016-5751

\section{Correspondence}

Peter A. Fasching, MD

Erlangen University Hospital, Department of Gynecology and Obstetrics, Comprehensive Cancer Center Erlangen EMN, Friedrich Alexander University of Erlangen-Nuremberg Universitätsstraße 21-23, 91054 Erlangen, Germany peter.fasching@uk-erlangen.de

丹 Deutsche Version unter:

https://doi.org/10.1055/s-0044-101614 


\section{ABSTRACT}

The treatment of metastatic breast cancer has become more complicated due to increasing numbers of new therapies which need to be tested. Therapies are now being developed to treat special clinical or molecular subgroups. Even though intrinsic molecular subtypes play a major role, more and more new therapies for subgroups and histological subtypes are being developed, such as the use of PARP inhibitors to treat patients with BRCA mutations (breast and ovarian cancer). Supportive therapies are also evolving, allowing problems such as alopecia or nausea and vomiting to be treated more effectively. Treatment-related side effects have a direct impact on the prognosis of patients with metastatic breast cancer, and supportive therapy can improve compliance. Digital tools could be useful to establish better patient management systems. This overview provides an insight into recent trials and how the findings could affect routine treatment. Current aspects of breast cancer prevention are also presented.

\section{Introduction}

Significant progress has been made in recent years in the treatment of metastatic breast cancer. The establishment of new targets and the introduction of new substance classes such as antibody-drug conjugates have significantly improved progressionfree survival rates and sometimes even the overall survival of some subgroups. Interest continues to focus on understanding how side effects occur and how they should be treated as well as on maintaining patients' quality of life. As it is becoming possible to describe personal risks more precisely, prevention is also becoming more individualized. The basic approaches in metastatic breast cancer, supportive therapies and prevention presented as part of new, recently published trials and at recent conferences (including the 2017 San Antonio Breast Cancer Symposium) are discussed in more detail below.

\section{Treatment of Metastatic HER2-positive and Triple-negative Breast Cancer (TNBC)}

\section{Data is consolidating on PARP inhibitors}

New targeted therapies for metastatic TNBC (mTNBC) are urgently needed to improve the prognosis of this patient population which has shown only a limited response to other lines of therapy. Several therapeutic approaches have recently been presented at conferences and in published articles.

\section{ZUSAMMENFASSUNG}

Die Behandlung des metastasierten Mammakarzinoms hat bei immer neu zu testenden Therapien deutlich an Komplexität zugenommen. Therapien werden nunmehr nur noch für spezielle klinische oder molekulare Subgruppen entwickelt. Hierbei spielen die intrinsischen, molekularen Subtypen zwar immer noch die größte Rolle, jedoch gibt es zunehmend auch Therapien, die subgruppen- oder sogar histologieübergreifend entwickelt werden, wie z.B. der PARP-Inhibitor bei BRCA-mutierten Patientinnen (Mamma- und Ovarialkarzinom). Aber auch Supportivtherapien entwickeln sich weiter, sodass Probleme wie die Alopezie besser behandelt werden können und neue Therapiearten von Übelkeit und Erbrechen etabliert werden. In einem engen Zusammenhang mit den Supportivtherapien stehen die Nebenwirkungen, welche bei Patientinnen mit einem metastasierten Mammakarzinom einen direkten Einfluss auf die Prognose haben. Hier könnten digitale Werkzeuge helfen, um ein besseres Patientinnenmanagement zu etablieren. Diese Übersichtsarbeit soll diese Aspekte vor dem Hintergrund neuer, aktuell publizierter Studien beleuchten und einen Einblick geben, wie sich diese Studien zu etablierten Routinetherapien verhalten. Zusätzlich werden aktuelle Aspekte der Mammakarzinomprävention beleuchtet.
Last year, it was reported that PARP inhibitors yielded promising results in the treatment of TNBC. In the OlympiAD trial, the PARP inhibitor olaparib showed a benefit with regard to progression-free survival in metastatic patients with proven germline mutations in the BRCA gene compared to selected chemotherapies (capecitabine, eribulin, vinorelbine) [1]. These results led to the drug being approved for use in the USA [2]. Patients with mTNBC especially benefitted. The EMBRACA trial presented data on the PARP inhibitor talazoparib [3], which was used in an almost identical setting as olaparib in the OlympiAD trial. Here too, progression-free survival (PFS) was significantly extended (8.6 vs. 5.6 months; HR 0.54 [0.41-0.71]; $\mathrm{p}<0.0001$ ). The objective rate of response was $63 \%$ and therefore more than double the rate for chemotherapies (27\%). Another study [4] investigated the effect of higher concentrations of talazoparib [5]. But higher systemic concentrations only resulted in more side effects but did not improve efficacy. It appears that the use of PARP inhibitors for TNBC is headed for success. It still unclear, however, whether a BRCA mutation is a precondition for this therapy.

\section{Other antibody-drug conjugates to treat mTNBC}

At the latest after the introduction of T-DM1, antibody-drug conjugates became a hot topic of discussion. Sacituzumab govitecan is an anti-Trop-2-SN-38 antibody-drug conjugate, which was used after second-line treatment in 110 patients with mTNBC until tumor progression or limiting toxicity [6]. The antibody is directed against the epithelial antigen Trop- 2 and is conjugated with the active metabolite of irinotecan. The objective response rate in this heavily treated patient cohort was $34 \%$ with a median duration of 
response of 7.6 months ( $95 \% \mathrm{Cl}: 4.8-11.3)$. The most important side effects were hematotoxicity (neutropenia grade 3/4: 41\%; febrile neutropenia: $8 \%$ ), fatigue and gastrointestinal symptoms. Sacituzumab govitecan appears to be an interesting drug which merits further research even if the response rates and side effects appear to be comparable to those of other monotherapies. A phase-III trial is already underway in the USA and another phaseIII trial is expected to kick-off in Europe in 2018.

\section{Immune checkpoint inhibitors headed for approval}

There is a growing body of data on immune checkpoint inhibitors. The pembrolizumab (anti-PD-1) and atezolizumab (anti-PD-L1) antibodies have already been tested in numerous studies on metastatic breast cancer, and the reported response rate was around $20 \%$ in patients pretreated for mTNBC [7]. Adams and colleagues recently presented results from the KEYNOTE-086 trial, where the first-line treatment consisted of monotherapy with pembrolizumab $(n=52)$. The response rate was $23 \%$ and the duration of response was 10.4 months. Patients who had partial or complete response (PR, CR) showed a significantly longer duration of response and a longer overall survival [8].

Numerous studies have investigated combinations of immune checkpoint inhibitors and chemotherapy or radiotherapy, and it is hoped that the number of patients who respond to this approach will increase. A phase-I/II study was recently presented which investigated a combination of eribulin mesylate and pembrolizumab in patients with mTNBC [9]. Irrespective of the PD-L1 status, a response rate of $26.4 \%$ was reported for the 107 patients treated in the study. If treatment was successful, the duration of response was 8.3 months, with more than half of patients responding for longer than 6 months.

Another study investigated a combination of pembrolizumab and trastuzumab in patients with trastuzumab-resistant advanced HER2-positive breast cancer [10]. Some level of response (15\%) was reported for PD-L1-expressing tumors but not for PD-L1-negative tumors. It appears therefore that HER2-pretreated tumors are not very immunogenic.

In summary, a clear trend has been observed with regard to the use of immune checkpoint inhibitors in breast cancer therapy: individual patients appear to respond very well and for an appreciable length of time to immunomodulatory therapy. Cytotoxic therapies might even achieve further improvements in the rate of response. But for the majority of patients, this therapy does not appear to result in any improvement. As neither positive nor negative predictive factors are known which could predict whether patients will respond, it is at present not possible to preselect patients. Further studies, particularly in the neoadjuvant setting, will determine whether the number and composition of tumor-infiltrating lymphocytes might play a role.

\section{Treatment of Hormone Receptor-positive, HER2-negative Advanced Breast Cancer}

\section{Data on CDK4/6 inhibitors in premenopausal patients}

In recent years, therapies for patients with metastatic, hormone receptor-positive and HER2-negative breast cancer have significantly improved, although a large percentage of patients still receive chemotherapy [11]. This could be changed by the introduction of therapies which are more effective than anti-hormone monotherapy. After the introduction of everolimus, interest has focused on CDK4/6 inhibitors. More and more studies in other patient cohorts are being published. Results from the MONALEESA-7 trial which investigated the CDK4/6 inhibitor ribociclib in a population of premenopausal patients were recently presented [12]. The trial compared an anti-endocrine therapy of choice (tamoxifen, letrozole or anastrozole) plus ovarian function suppression (OFS) with the same therapy plus the addition of ribociclib. The median progression-free survival was 23.8 months in the ribociclib arm compared with 13 months in the placebo arm ( $\bullet$ Fig. 1). The overall response rate was significantly higher in patients with a measurable lesion at the start of therapy in the ribociclib arm compared with the placebo arm (51\% vs. $36 \%$ ). Grade $3 / 4$ neutropenia was reported in $61 \%$ of patients in the ribociclib arm, although this side effect was not symptomatic in most patients. Patients in the ribociclib arm additionally benefited from a prolongation of the time to deterioration of their quality of life. This combination hormone therapy should therefore be recommended to treat premenopausal high-risk patients.

\section{Studies with CDK4/6 inhibitors are combined to answer further questions}

The American regulatory authority (FDA) recently presented an analysis in which all studies which filed for approval of a CDK4/6 inhibitor were pooled for analysis [13]. This included studies on palbociclib, ribociclib and abemaciclib in combination with an aromatase inhibitor as the initial therapy for metastatic or inoperable breast cancer. The intention-to-treat population included 1992 patients. Progression-free survival was compared for patients aged $\geq 70$ years and patients aged $<70$ years. The progressionfree survival benefit from CDK4/6 inhibitors was similar for both groups of patients. The side effects were only slightly higher in older patients. The conclusion is that higher age is no reason to refuse therapy with CDK4/6 inhibitors to older patients.

Another pooled analysis combined the datasets of the MONARCH-2 and MONARCH-3 trials to look for predictors which could be used to identify patients who would respond particularly well to therapy with abemaciclib and aromatase inhibitors [14]. Out of all the univariate analyses analyzing progression-free survival, only the interval between primary treatment and the start of therapy was found to be a predictor for the efficacy of abemaciclib in the setting of advanced cancer. The number of previous endocrine therapies, previous endocrine therapy for advanced disease, the time from diagnosis to metastasis, and primary metastatic status were not predictors for the efficacy of abemaciclib. Howev- 


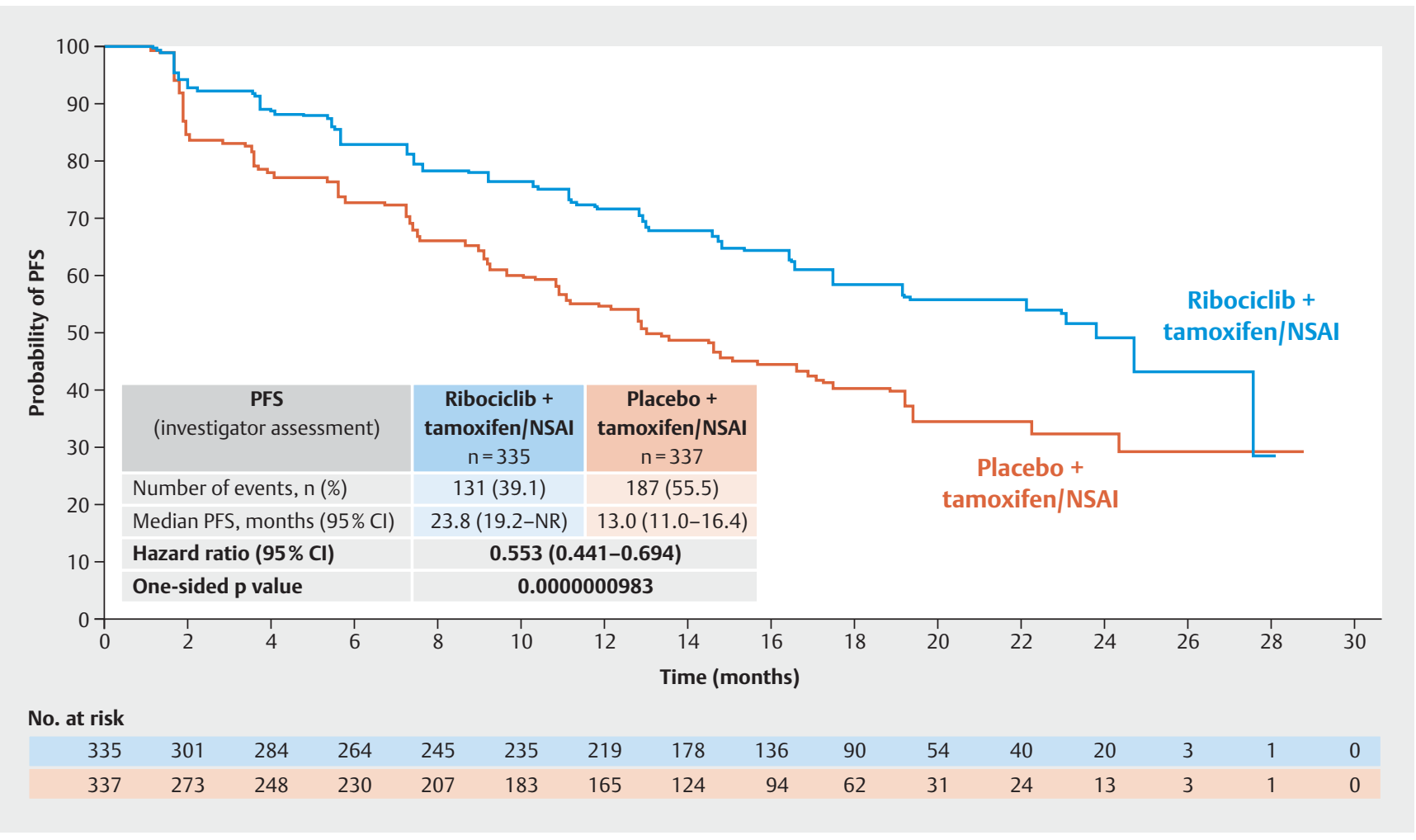

- Fig. 1 Progression-free survival (response determined by the examiner) in the MONALEESA trials. Goserelin was administered in both arms. NR: not achieved, Cl: confidence interval (based on [12]).

er, no confidence intervals were presented in this analysis, which limits the interpretation of the data.

\section{Establishment of new mTOR inhibitors unsuccessful}

The current therapy recommendations and guidelines $[15,16]$ recommend using three different endocrine therapies in the metastatic setting. One of these therapies includes the mTOR inhibitor everolimus. Vistusertib is a new dual mTOR inhibitor which has been tested in a single study. Vistusertib plus fulvestrant was compared with a therapy consisting of everolimus and fulvestrant [17]. Progression-free survival was longest (12.3 months) following treatment with everolimus and fulvestrant. Both therapy arms with vistusertib were inferior.

\section{Androgen receptor inhibition attempts to prove its worth}

The androgen receptor inhibitor enzalutamide has already shown some benefits in the treatment of TNBC [18-20]. Data on the efficacy of this therapy to treat hormone receptor-positive breast cancer have now also been presented [21]. Therapy with enzalutamide and exemestane was compared with therapy using exemestane alone in patients with advanced breast cancer with and without previous endocrine therapy. No difference between the therapy arms was reported for the overall patient population. However, when analysis was limited to a subgroup in which gene expression testing had detected an activation of the androgen receptor pathway, a significant benefit was detected for patients who had not had prior treatment (hazard ratio [HR]: 0.44; PFS 16.5 vs. 4.3 months). Enzalutamide was even found to benefit patients with prior treatment (HR: 0.55; PFS: 6 vs. 4.3 months). This therapy merits investigation in further studies.

\section{Supportive Therapy}

Supportive therapies are playing an increasingly important role in oncology and are being investigated in numerous scientific studies. The often significant side effects of modern multimodal therapies can only be controlled by optimal supportive treatment, and supportive therapy is needed to improve the quality of life and long-term compliance of patients.

\section{Benefits of acupuncture and physical exercise in therapy-induced arthralgia}

The problem of compliance is still unresolved, with high numbers of patients terminating their adjuvant endocrine treatment because of side effects such as arthralgia [22-25]. Acupuncture is an alternative, non-drug therapy for arthralgia. However, the data is inconsistent. A study was carried out to compare the use of acupuncture with feigned acupuncture (short needles at non-acupuncture sites) and a control group over a period of 12 weeks (first 6 weeks: $2 \times$ per week, subsequently $1 \times$ per week) [26]. A total of 226 patients with breast cancer who reported $\geq 3$ points on the pain scale (Brief Pain Inventory, BPI) at the start of treatment with an aromatase inhibitor (AI) or who reported increasing 
pain during Al therapy were included in the study. Acupuncture was carried out in 11 centers by licensed therapists who had received extensive training. Multiple measurements showed significant improvements in the strongest level of pain after 6 weeks (primary endpoint) compared to the feigned acupuncture group $(p=0.01)$ and the control group who received no form of acupuncture intervention $(p=0.01)$. Significant improvements were also reported with regard to average levels of pain and joint stiffness. The benefit persisted for 12 weeks after the acupuncture intervention.

Another study investigated the effect of physical exercise on arthralgia due to Al therapy [27]. A significant improvement in pain scores was reported after 12 months of exercise $(p=0.067)$ if patients followed a program of 120-150 minutes of walking or running per week and complied with more than $70 \%$ of the planned program. This also positively affected patients' compliance with Al therapy $(p=0.03)$.

\section{Diarrhea control depends on the treating physician}

Neratinib, a pan-Her tyrosine kinase inhibitor, is another oral adjuvant therapy for patients with HER-2-positive breast cancer who have already had adjuvant therapy with trastuzumab. However, neratinib requires optimal supportive therapy. The rate of grade III diarrhea reported in the approval study was $39.8 \%$, which led to termination of the therapy by $16.8 \%$ of patients. The CONTROL study compared an intensified loperamide regimen $(12 \mathrm{mg} /$ day, $\mathrm{d} 1-14$, followed by $6-8 \mathrm{mg} /$ day, $\mathrm{d} 15-56)$ with combinations of loperamide and the steroid budesonide or the cholesterol-lowering drug colestipol [28]. Grade III diarrhea occurred in 30.7, 25.0 and $7.7 \%$ of patients, and therapy termination rates were 20.4 , 9.4 and $0 \%$, respectively. The addition of budesonide or colestipol appears to reduce the duration and number of diarrhea episodes and could therefore improve compliance with neratinib therapy. The final analysis of the CONTROL study will be carried out when all patients have completed the 12-month neratinib therapy.

\section{Alopecia from chemotherapy partially avoidable}

Alopecia is an extremely onerous side effect of chemotherapy. Four studies investigated the efficacy of scalp cooling during therapy. The success rate during treatment with anthracyclines was 50-62.5\% [29, 30]. Menopausal status, therapy setting (neoadjuvant versus adjuvant) and dose density had no impact on alopecia rates. The problem is the high rate of around $30 \%$ of patients who discontinue scalp cooling because of the pain, with about half of these patients subsequently losing all of their hair [29]. It should be noted, however, that grade I loss of hair after scalp cooling was also rated as a success in the studies. Grade I is a loss of hair of up to $50 \%$, which can also represent a significant burden for patients.

\section{Nausea and vomiting rates continue to fall}

Although there are excellent antiemetic drugs and standards, there is still potential for improvement. The use of aprepitant is standard during treatment with anthracyclines, but its use in moderately emetogenic chemotherapies has not been investigated much. A randomized, double-blinded phase-III trial which compared fosaprepitant combined with ondansetron and dexamethasone, d1, with ondansetron, d1-3, and dexamethasone, d1, in 231 patients with breast cancer showed a complete response (no vomiting or need of rescue medication during the trial) in 76.4 vs. $68.6 \%$ of patients [31]. Another multicenter study investigated a combination of 5-HT3 and NK1-receptor antagonists in a combined capsule (NEPA) to simplify therapy. The interim analysis presented the results for 2384 patients receiving highly or moderately emetogenic chemotherapy (HEC/MEC) [32]. As regards the primary endpoint, over $90 \%$ of the study participants who received HEC or MEC reported that their daily life and quality of life was not affected by vomiting. The rate of complete response (no nausea, no rescue medication) was $74 \%$. Efficacy was rated by both the physicians and the study participants as good to very good in more than $90 \%$ of cases.

\section{Neuropathy continues to be a big problem}

One of the biggest current challenges is chemotherapy-related neuropathy, particularly over the longer term. A study of 238 patients in the adjuvant setting and 442 patients with metastasis showed that $30 \%$ of all studied patients were significantly or strongly affected by neuropathies in their daily life [33]. Neuropathies were significantly associated with sleep disorders, pain, impairments of physical function, restricted social life, fatigue, anxiety and depression. $32 \%$ had not received any coping strategies from their treating physician. The lack of counseling with regard to supportive therapies was associated with a significantly higher negative impact.

The above-mentioned studies on supportive therapies clearly illustrate the importance of a holistic approach for patients and the importance of targeting potential side effects both prophylactically and therapeutically.

\section{Bone Oncology}

The preventive effect of bisphosphonates on bone metabolism and bone density and their contribution to reducing the risk of fractures has been known since many years. An Oxford meta-analysis once again showed that the adjuvant use of bisphosphonates to prevent osteoporosis after menopause additionally resulted in a $34 \%$ improvement in breast cancer-specific disease-free survival (DFS) and even a 17\% improvement in overall survival [34]. This has led to a positive recommendation for adjuvant bisphosphonate therapy in parallel to treatment $\mathrm{Al}$ in the AGO and the current DGGG S-3 guidelines $[15,16]$.

\section{Duration of adjuvant bisphosphonate therapy}

But the question remains how long the respective bisphosphonate therapy should be administered. In this context, the SUCCESS study investigated the impact of 2 years' $(4 \mathrm{mg}$ zoledronic acid every 3 months) vs. 5 years' ( 4 mg zoledronic acid every 3 months for 2 years, then every 6 months) bisphosphonate therapy on DFS and overall survival (OS) ( $\triangleright$ Fig. 2) [35]. The results for 2802 patients out of the original 3754 patients were included in the analysis. The two study groups showed no significant differences with regard to their basic characteristics or tumor characteristics. The maximum observation period was 4 years (the median observation period was 2.95 years for DFS and 3 years for OS). DFS and OS were defined based on the STEEP criteria. The results of the 

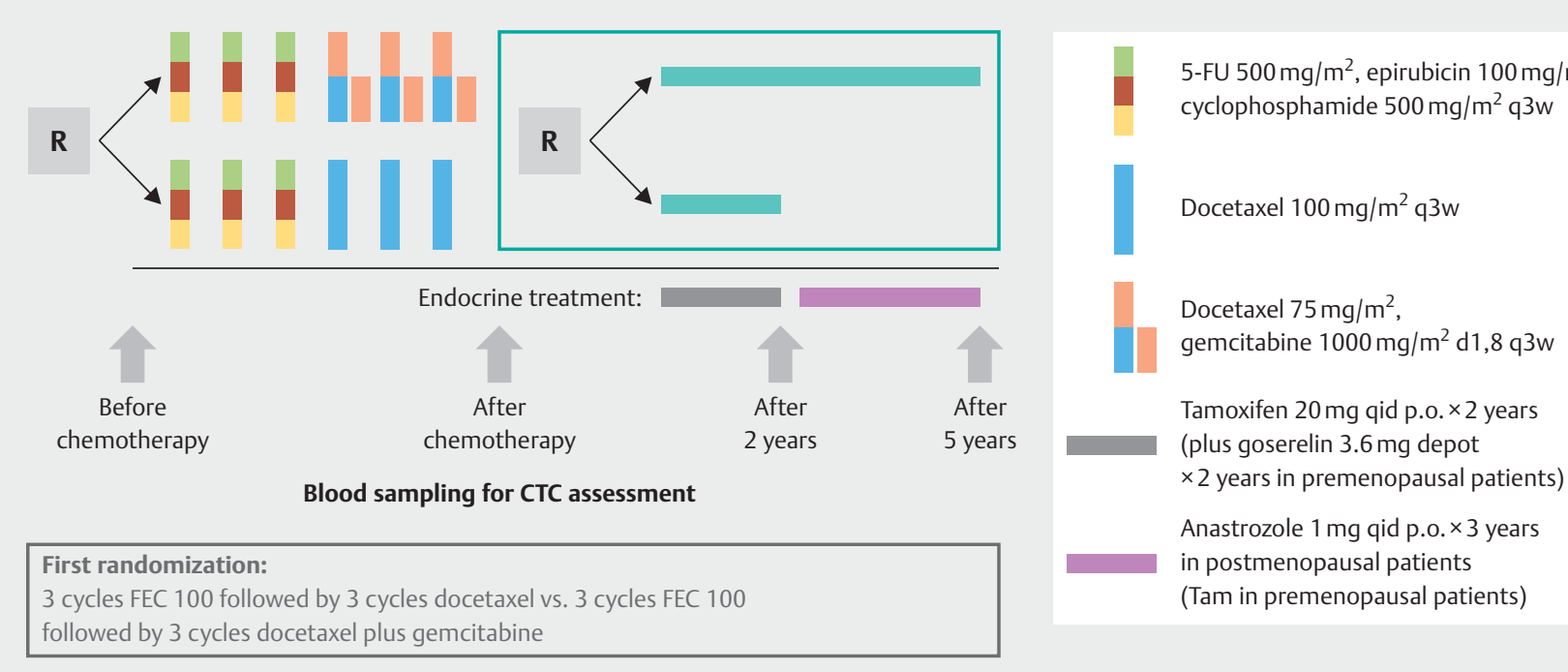

Second randomization:

5 years vs. 2 years of zoledronate

( $4 \mathrm{mg}$ i.v. every 3 months for 2 years, followed by $4 \mathrm{mg}$ i.v. every 6 months for 3 years

vs. $4 \mathrm{mg}$ i.v. every 3 months for 2 years)

- Fig. 2 SUCCESS A study design (based on [35]).

analysis showed no significant differences in terms of DFS $(p>0.827)$ or OS ( $p>0.713$ ) between the 2-year and the 5-year bisphosphonate therapy [35]. Multiple Cox regression analysis adjusted for a number of risk factors also showed no significant differences between groups with regard to DFS, bone recurrence or OS in either premenopausal or postmenopausal women with breast cancer. No differences were found between the two groups with regard to circulating tumor cells (CTC). The rate of side effects was significantly higher after 5 years of treatment compared to 2 years of bisphosphonate therapy. Osteonecrosis of the jaw (ONJ) occurred in 11 and 5 cases, respectively, during 5-year or 2-year bisphosphonate therapy. The limitations of the study listed by the authors included the relatively short observation period, the unbalanced rate of "loss to follow up" and the relatively rare events. Unfortunately this study also lacked a control group which did not receive bisphosphonate therapy [35].

\section{Aromatase inhibitors must be monitored continuously for bone protection}

As part of the ABCSG-16 trial, the impact of extending adjuvant endocrine therapy (EAT) with anastrozole by a further 2 vs. 5 years after 4-6 years of primary endocrine therapy was investigated. As regards the primary endpoint of disease-free survival, the report [36] found that after 10 years, DFS for the group which had two additional years aromatase inhibitor therapy was $71.1 \%$, while DFS for the group with an additional 5 years was $70.3 \%$ and therefore not significantly different (HR: $0.997 ; 95 \% \mathrm{Cl}$ : $0.86-1.15$; $\mathrm{p}=0.982$ ). As regards the side effect "fractures" the group with 5 years of EAT had a higher rate of fractures compared to the group which received 2 years of anastrozole therapy ( $\boldsymbol{F i g . 3}$ )
[36]. The figures were not adjusted for potential bone protective therapies.

\section{Molecular pathways of breast cancer risk, bone metabolism and breast development are linked}

It has been known for some time that the signaling pathways which regulate bone metabolism (RANKL/RANK/OPG) also regulate progesterone-based proliferation in the breast and can have an impact on the pathogenesis and progression of breast cancer [37-39]. It was recently reported that this signaling pathway is particularly important in patients with a BRCA 1 mutation. RANKL appears to be the main mediator in the pathogenesis of breast cancer in this patient population [40-42], meaning that a blockade of this signaling pathway might be a useful strategy for breast cancer prevention. A study with denosumab is planned to investigate this point further [42]. Other substances such as the selective progesterone receptor modulator telapristone may also be of interest, as preclinical models have shown that these substances inhibit the paracrine expression of RANKL [43].

\section{Quality of Life and Digitization of Medicine}

Quality of life, therapies with few side effects and survival are among the most important therapy goals for patients [44,45], and patient well-being, therapy, compliance and overall wellbeing are mutually dependent. Side effects, e.g. due to therapy with aromatase inhibitors, can result in the patient terminating their therapy [22-25], which in turn is likely to result in a poorer prognosis [46]. This means that taking the patient's quality of life 


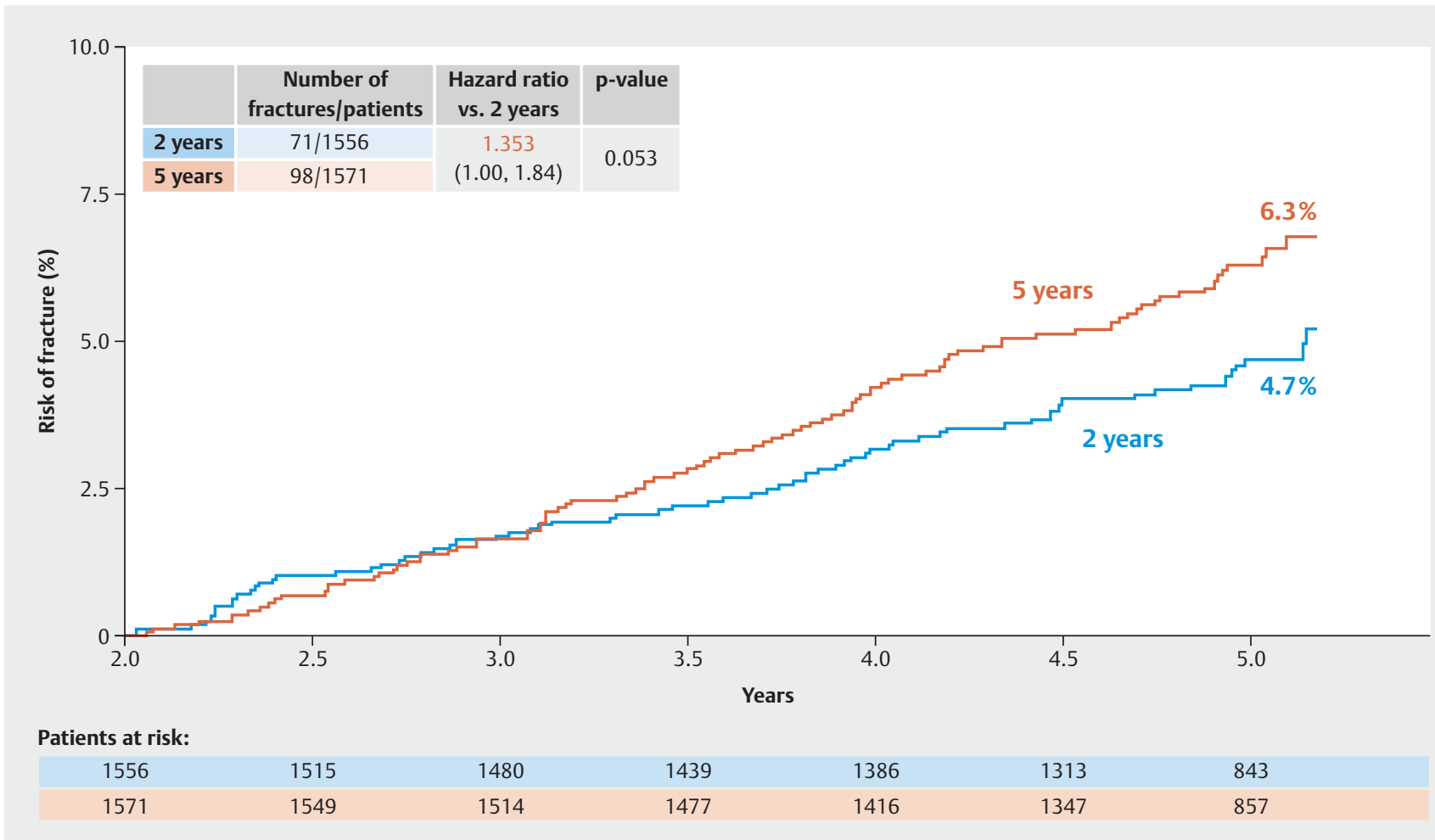

Fig. 3 Fracture rates in the ABCSG-16 trial. (2 years: 2 years of therapy with an aromatase inhibitor; 5 years: 5 years of therapy with an aromatase inhibitor) (based on [36]).

into consideration is also important in the context of improving her prognosis.

\section{Digital medicine to improve communication, quality of life and prognosis}

Digitization in medicine is constantly increasing [47]. There are still huge opportunities to optimize the healthcare for patients by improving therapy selection, patient care and the management of related areas. Much has been written in this context about "big and smart data". Given the rapid advances in machine learning and the interoperability of databases (which has done much to simplify their use), it is expected that central patient care processes will be automated [47] and electronic tools will increasingly be used to communicate with patients $[11,48]$. But it will also be necessary to ensure that interests of the patients (data confidentiality, self-determination and data sovereignty) are maintained [49].

The field has huge potential and presents many opportunities. To take just one example, it has been shown that the use of electronic tools to provide a constant line of communication between the treating medical staff and patients as a means of monitoring side effects can result in better overall survival [50]. The integration of such platforms in various studies across the Germanspeaking world is increasing. Electronic patient-reported outcome systems have already been tested since several years in studies such as PRAEGNANT [51, 52], Seraphina [53] and Precycle [54].

\section{Knowledge processing and knowledge exchange for physicians}

As the breadth of knowledge continues to grow by leaps and bounds, it cannot be ruled out that in the long run knowledge will be permanently managed using digital means. The App "Mammakarzinom Transparent" (https://mammakarzinom.onkowissen. $\mathrm{de} /[55])$ is just one attempt to make therapy algorithms available and include additional information on efficacy and side effects. Examples of clickable icons are shown in - Figs. 4 to 6; clicking on the icon will call up further information about the respective therapy.

\section{Quality of life in the metastatic setting}

In Germany, quality of life is an accepted endpoint in addition to overall survival for the assessment of therapies after they have been approved. In recent evaluations, progression-free survival was not accepted for assessments about the additional benefit of a specific therapy. However, it is well known that for the patient disease progression has a negative impact on their quality of life and a delay in disease progression will therefore improve patients' quality of life $[56,57]$. Particularly in the metastatic therapy setting where there are frequent changes of therapy, data about the extent to which therapy sequences influence the further course of disease and quality of life have not been thoroughly investigated. New studies which take a comprehensive look at therapy lines [51, 58] could help to collect new data and improve the care of these patients. 


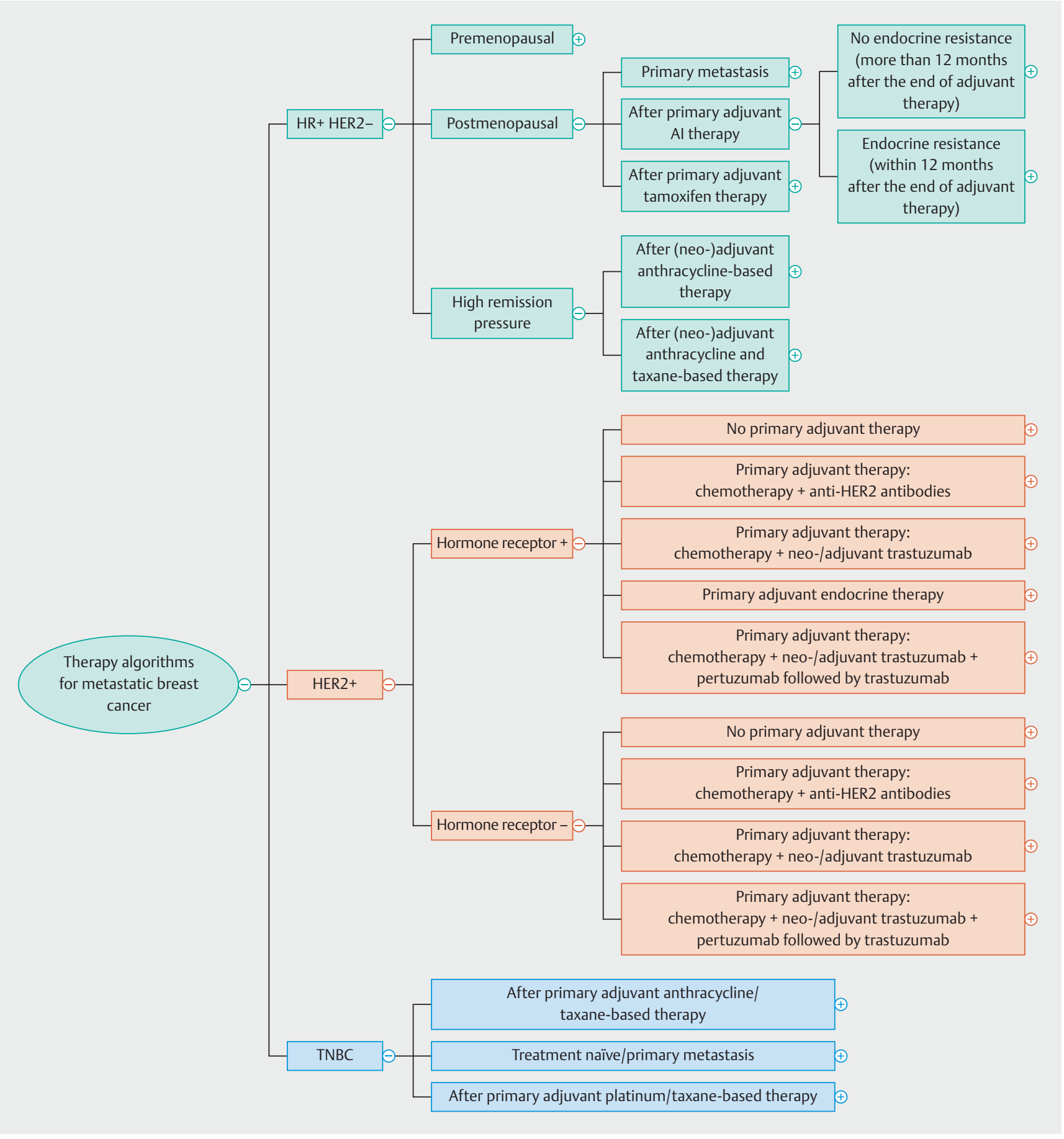

- Fig. 4 Current therapy algorithms for metastatic breast cancer (schematic) (based on [55]).

\section{Prevention}

\section{Importance of panel genes}

In the last few years, the medical understanding of genetic and non-genetic risk factors has significantly improved $[59,60]$. In addition to the BRCA 1 and BRCA 2 genes, the clinical importance of socalled panel genes (e.g., the following genes which are associated with a high or moderate risk of breast cancer: CHEK2, PALB2, ATM,
RAD51D, BARD1, MSH6 and others) is being investigated [61 -67]. One study reported that PALB2 (OR: 7.5) might need to be classified as belonging to the group of high-risk genes while ATM, BARD1 and RAD51D could probably be classified as moderate risk genes [62]. No increased risk was reported for the genes BRIP1, RAD51C, MRE11A, RAD50, NBN, MLH1 and PMS2 [62]. It is not yet clear to what extent this information will have an impact on prevention. 


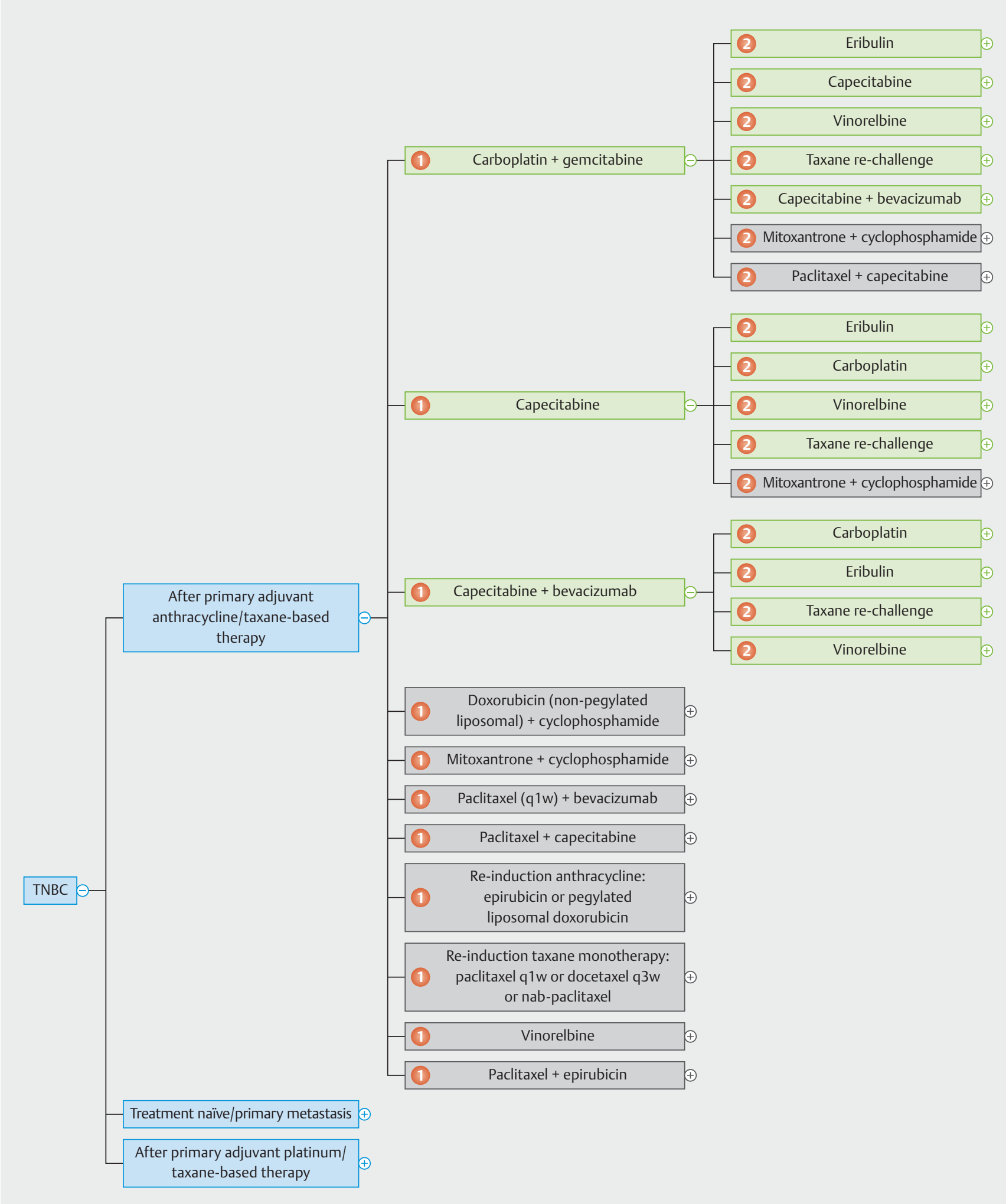

- Fig. 5 Current therapy algorithms for patients with metastatic triple-negative breast cancer (schematic) (based on [55]). 


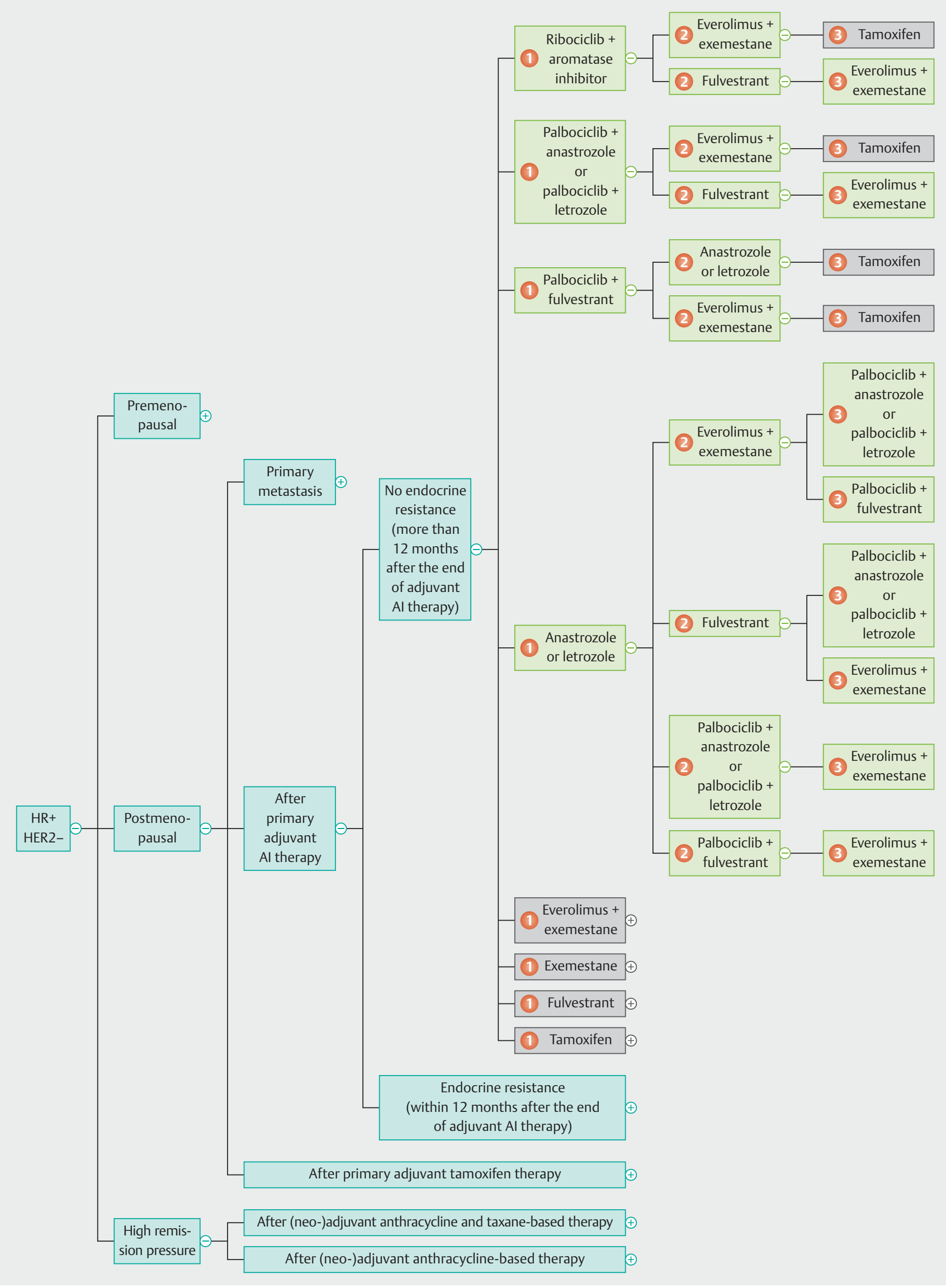

D Fig. 6 Current therapy algorithms for patients with hormone receptor-positive, HER2-negative breast cancer (schematic) (based on [55]). 


\section{Which population is suitable for mutation testing?}

The approval of the first PARP inhibitor to treat metastatic breast cancer in the USA [2] raised the question whether genotyping should be carried out in all patients who could be considered for this therapy. A recent study presented the mutation frequencies for BRCA 1 and BRCA 2 and other panel genes irrespective of the usual test criteria used in predictive genetic diagnostics [68] (see chapter "Prognostic and Predictive Factors). The question about the extent to which predictive genetic testing could and should be expanded also includes the debate about whether patients with low mutation rates needed to be informed about any variants of unclear significance (VUS) detected during testing. In the case of genetic changes where not much is known about their clinical relevance, the uncertainty could lead to anxiety, worry and even unjustified measures. At present, more than 3000 of such VUS in the BRCA 1 and BRCA 2 genes are known. Significant progress has also been achieved recently in this area. Studies have shown that functional in vitro analysis of 139 VUS in BRCA 2 was able to identify 13 more pathogenic mutations, while 12 could be classified as harmless variants [69]. More analyses of this type could significantly decrease the diagnostic uncertainty.

\section{Importance of low-penetrance risk genes}

In addition to high and moderate risk genes, the importance of common genetic changes with significantly lower risk modifications is becoming increasingly clear. A further 75 variants have just been recently validated $[70,71]$. Other variants and their clinical importance are also gradually being characterized [72-76]. The data obtained could be summarized in scores and tested in clinical practice [77]. Combinations with clinical risk factors such as mammographic density, size or BMI could also be of clinical use [78-82]. However, such studies still need to be carried out.

\section{Acknowledgements}

This work was partly the result of funding received from Riemser and the PRAEGNANT study network, neither of which had any involvement in the compilation of this manuscript. The authors alone are responsible for the contents of this paper.

\section{Conflict of Interest}

A. D. H. received honoraria from AstraZeneca, Genomic Health, Roche, Novartis, Celgene and Pfizer. N. N. received consultancy honoraria from Janssen-Cilag and travel support from Novartis. F. O. received speaker and consultancy honoraria from Amgen, Celgene, TEVA, AstraZeneca, Novartis, Roche, and MSD. F.-A. T. received honoraria from Astra Zeneca, Genomic Health and Novartis. H.-C. K. received honoraria from Carl Zeiss meditec, TEVA, Theraclion, Novartis, Amgen, Astra Zeneca, Pfizer, Janssen-Cilag, GSK, LIV Pharma, Roche and Genomic Health. P. H. received honoraria, unrestricted educational grants and research funding from Amgen, AstraZeneca, Eli Lilly, MSD, Novartis, Pfizer and Roche. P. A. F. received honoraria from Roche, Pfizer, Novartis and Celgene. His institution conducts research for Novartis. H. T. received honoraria from Novartis, Roche, Celgene, TEVA, Pfizer and travel support from Roche, Celgene and Pfizer. J. E. received honoraria from Roche, Celgene, Novartis, Pfizer, Pierre Fabre, TEVA and travel support from Celgene, Pfizer, TEVA and Pierre Fabre. M. P. L. has participated on advisory boards for AstraZeneca, MSD, Novartis, Pfizer, Genomic Health and Roche and has received honoraria for lectures from Lilly, Roche, Novartis, Pfizer, Genomic
Health, AstraZeneca, medac and Eisai. M. W. received speaker honoraria from Astra Zeneca, Celgene and Novartis. V. M. received speaker honoraria from Amgen, Astra Zeneca, Celgene, Daiichi-Sankyo, Eisai, Pfizer, Pierre-Fabre, Novartis, Roche, Teva, Janssen-Cilag and consultancy honoraria from Genomic Health, Roche, Pierre Fabre, Amgen, Daiichi-Sankyo and Eisai. E. B. received honoraria from Novartis, Riemser and Hexal for consulting and clinical research management activities. A. S. received honoraria from Roche, Celgene, AstraZeneca, Novartis, Pfizer, Zuckschwerdt Verlag GmbH, Georg Thieme Verlag, Aurikamed GmbH, MCI Deutschland $\mathrm{GmbH}$, bsh medical communications $\mathrm{GmbH}$ and promedicis $\mathrm{GmbH}$. W.J. received honoraria and research grants from Novartis, Roche, Pfizer, Lilly, AstraZeneca, Chugai, Sanofi, Daichi, Tesaro. F. S. participated on advisory boards for Novartis, Amgen and Roche and received honoraria for lectures from Roche, Novartis and Pfizer.

\section{References}

[1] Robson M, Im S-A, Senkus E et al. Olaparib for metastatic breast cancer in patients with a germline BRCA mutation. N Engl J Med 2017; 377 : 523-533

[2] United States Food and Drug Administration (FDA). FDA approves first treatment for breast cancer with a certain inherited genetic mutation (2018). Online: https://wwwfdagov/NewsEvents/Newsroom/ PressAnnouncements/ucm592347.htm; last access: 16.01.2018

[3] Litton J, Rugo H, Ettl J et al. Abstract GS6-07: EMBRACA: A phase 3 trial comparing talazoparib, an oral PARP inhibitor, to physician's choice of therapy in patients with advanced breast cancer and a germline BRCA mutation. Cancer Res 2018; 78: GS6-07-GS06-07

[4] Turner NC, Telli ML, Rugo HS et al. Final results of a phase 2 study of talazoparib (TALA) following platinum or multiple cytotoxic regimens in advanced breast cancer patients (pts) with germline BRCA1/2 mutations (ABRAZO). J Clin Oncol 2017; 35 (Suppl.): Abstr. 1007

[5] Telli M, Turner N, Mailliez A et al. Abstract P1-14-03: ABRAZO: Exposureefficacy and -safety analyses of breast cancer patients with germline BRCA1/2 mutations receiving talazoparib in a phase 2 open-label trial. Cancer Res 2018; 78: P1-14-03-P11-14-03

[6] Bardia A, Vahdat L, Diamond J et al. Abstract GS1-07: Sacituzumab govitecan (IMMU-132), an anti-Trop-2-SN-38 antibody-drug conjugate, as $\geq 3$-line therapeutic option for patients with relapsed/refractory metastatic triple-negative breast cancer (mTNBC): efficacy results. Cancer Res 2018; 78: GS1-07-GS01-07

[7] Nanda R, Chow LQ, Dees EC et al. Pembrolizumab in patients with advanced triple-negative breast cancer: phase Ib KEYNOTE-012 study. J Clin Oncol 2016; 34: 2460-2467

[8] Adams S, Loi S, Toppmeyer D et al. Abstract PD6-10: KEYNOTE-086 cohort B: Pembrolizumab monotherapy for PD-L1-positive, previously untreated, metastatic triple-negative breast cancer (mTNBC). Cancer Res 2018; 78: PD6-10-PD16-10

[9] Tolaney S, Kalinsky K, Kaklamani V et al. Abstract PD6-13: Phase 1b/2 study to evaluate eribulin mesylate in combination with pembrolizumab in patients with metastatic triple-negative breast cancer. Cancer Res 2018; 78: PD6-13-PD16-13

[10] Loi S, Giobbe-Hurder A, Gombos A et al. Abstract GS2-06: Phase Ib/II study evaluating safety and efficacy of pembrolizumab and trastuzumab in patients with trastuzumab-resistant HER2-positive metastatic breast cancer: results from the PANACEA (IBCSG 45-13/BIG 4-13/KEYNOTE014) study. Cancer Res 2018; 78: GS2-06-GS02-06

[11] Hartkopf AD, Graf J, Simoes E et al. Electronic-based patient-reported outcomes: willingness, needs, and barriers in adjuvant and metastatic breast cancer patients. JMIR Cancer 2017; 3: e11 
[12] Tripathy D, Sohn J, Im S-A et al. Abstract GS2-05: First-line ribociclib vs placebo with goserelin and tamoxifen or a non-steroidal aromatase inhibitor in premenopausal women with hormone receptor-positive, HER2-negative advanced breast cancer: results from the randomized phase III MONALEESA-7 trial. Cancer Res 2018; 78: GS2-05-GS02-05

[13] Singh H, Howie L, Bloomquist E et al. Abstract GS5-06: A U.S. food and drug administration pooled analysis of outcomes of older women with hormone-receptor positive metastatic breast cancer treated with a CDK4/6 inhibitor as initial endocrine based therapy. Cancer Res 2018; 78: GS5-06-GS05-06

[14] Goetz M, O'Shaughnessy J, Sledge G et al. Abstract GS6-02: The benefit of abemaciclib in prognostic subgroups: an exploratory analysis of combined data from the MONARCH 2 and 3 studies. Cancer Res 2018; 78: GS6-02-GS06-02

[15] Kommission Mamma der Arbeitsgemeinschaft Gynäkologische Onkologie e.V. in der Deutschen Gesellschaft für Gynäkologie und Geburtshilfe e.V. sowie in der Deutschen Krebsgesellschaft e.V. Diagnostik und Therapie von Patientinnen mit primärem und metastasiertem Brustkrebs (2017). Online: https://www.ago-online.de/fileadmin/downloads/ leitlinien/mamma/2017-03/AGO_deutsch/PDF_Gesamtdatei_deutsch/ Alle_aktuellen_Empfehlungen_2017.pdf; last access: 07.01.2018

[16] Leitlinienprogramm Onkologie (Deutsche Krebsgesellschaft Deutsche Krebshilfe AWMF). S3-Leitlinie Früherkennung, Diagnose, Therapie und Nachsorge des Mammakarzinoms, Version 4.0, 2017 AWMF Registernummer: 032-045OL. 2017. Online: http://www.leitlinienprogrammonkologie.de/leitlinien/mammakarzinom/; last access: 07.01.2018

[17] Schmid P, Zaiss M, Harper-Wynne C et al. Abstract GS2-07: MANTA - A randomized phase II study of fulvestrant in combination with the dual mTOR inhibitor AZD2014 or everolimus or fulvestrant alone in estrogen receptor-positive advanced or metastatic breast cancer. Cancer Res 2018; 78: GS2-07-GS02-07

[18] Traina TA, O’Shaughnessy J, Nanda R et al. Preliminary results from a phase 2 single-arm study of enzalutamide, an androgen receptor (AR) inhibitor, in advanced AR plus triple-negative breast cancer (TNBC). Cancer Res 2015; 75: Abstract P5-19-09. doi:10.1158/1538-7445.SABCS14-P519-09

[19] Traina TA, Miller K, Yardley DA et al. Results from a phase 2 study of enzalutamide (ENZA), an androgen receptor (AR) inhibitor, in advanced AR plus triplenegative breast cancer (TNBC). J Clin Oncol 2015; 33 (15_suppl): 1003-1003. doi:10.1200/jco.2015.33.15_suppl.1003

[20] Cortes ], Crown ], Awada A et al. Overall survival (OS) from the phase 2 study of enzalutamide (ENZA), an androgen receptor (AR) signaling inhibitor, in AR plus advanced triple-negative breast cancer (aTNBC). Eur J Cancer 2015; 51: S265-S265

[21] Krop I, Abramson V, Colleoni M et al. Abstract GS4-07: Results from a randomized placebo-controlled phase 2 trial evaluating exemestane \pm enzalutamide in patients with hormone receptor-positive breast cancer. Cancer Res 2018; 78: GS4-07-GS04-07

[22] Nabieva N, Kellner S, Fehm T et al. Patient and tumor characteristics and their influence on early therapy persistence with letrozole in postmenopausal patients with early breast cancer. Ann Oncol 2017. doi:10.1093/ annonc/mdx630

[23] Hadji P, Jackisch C, Bolten W et al. COMPliance and Arthralgia in Clinical Therapy: the COMPACT trial, assessing the incidence of arthralgia, and compliance within the first year of adjuvant anastrozole therapy. Ann Oncol 2014; 25: 372-377

[24] Hadji P, Ziller V, Kyvernitakis ] et al. Persistence in patients with breast cancer treated with tamoxifen or aromatase inhibitors: a retrospective database analysis. Breast Cancer Res Treat 2013; 138: 185-191

[25] Hadji P, Blettner M, Harbeck N et al. The Patient's Anastrozole Compliance to Therapy (PACT) Program: a randomized, in-practice study on the impact of a standardized information program on persistence and compliance to adjuvant endocrine therapy in postmenopausal women with early breast cancer. Ann Oncol 2013; 24: 1505-1512
[26] Hershman D, Unger ], Greenlee H et al. Abstract GS4-04: Randomized blinded sham- and waitlist-controlled trial of acupuncture for joint symptoms related to aromatase inhibitors in women with early stage breast cancer (S1200). Cancer Res 2018; 78: GS4-04-GS04-04

[27] Tamaki K, Takaesu M, Nagamine S et al. Abstract P6-11-01: Final results of the randomized trial of exercise intervention vs. usual care for breast cancer patients with aromatase inhibitor to prevent and improve the aromatase inhibitor induced arthralgia. Cancer Res 2018; 78: P6-11-01P16-11-01

[28] Hurvitz S, Chan A, lannotti N et al. Abstract P3-14-01: Effects of adding budesonide or colestipol to loperamide prophylaxis on neratinib-associated diarrhea in patients with HER2+ early-stage breast cancer: the CONTROL trial. Cancer Res 2018; 78: P3-14-01-P13-14-01

[29] Silva G, Moreira R, Gimenes D et al. Abstract P6-11-06: Efficacy of scalp cooling in preventing chemotherapy-induced alopecia in breast cancer patients: a retrospective, comprehensive review of 330 cases of Brazil. Cancer Res 2018; 78: P6-11-06-P16-11-06

[30] Kurbacher C, Kurbacher A, Herz S et al. Abstract P6-11-14: Safety and effectiveness of sensor-controlled scalp cooling to prevent alopecia in primary breast cancer patients receiving neoadjuvant or adjuvant epirubicin, taxanes, or both. Cancer Res 2018; 78: P6-11-14-P16-11-14

[31] Weinstein C, Jordan K, Green S et al. Abstract P6-11-11: Single-dose fosaprepitant for the prevention of chemotherapy-induced nausea and vomiting in patients with breast cancer receiving moderately emetogenic chemotherapy regimens. Cancer Res 2018; 78: P6-11-11-P16-11-11

[32] Schilling J, Klare P, Heilmann V et al. Abstract P6-11-05: NEPA for CINV prevention in highly or moderately emetogenic chemotherapy - interim results of a German non-interventional study on quality of life and efficacy. Cancer Res 2018; 78: P6-11-05-P16-11-05

[33] Zaleta A, Miller M, Johnson J et al. Abstract P6-11-08: Chemotherapyinduced peripheral neuropathy and quality of life among breast cancer survivors. Cancer Res 2018; 78: P6-11-08-P16-11-08

[34] Early Breast Cancer Trialists' Collaborative Group. Adjuvant bisphosphonate treatment in early breast cancer: meta-analyses of individual patient data from randomised trials. Lancet 2015; 386: 1353-1361

[35] Janni W, Friedl T, Fehm T et al. Abstract GS1-06: Extended adjuvant bisphosphonate treatment over five years in early breast cancer does not improve disease-free and overall survival compared to two years of treatment: phase III data from the SUCCESS A study. Cancer Res 2018; 78: GS1-06-GS01-06

[36] Gnant M, Steger G, Greil R et al. Abstract GS3-01: A prospective randomized multi-center phase-III trial of additional 2 versus additional 5 years of anastrozole after initial 5 years of adjuvant endocrine therapy - results from 3,484 postmenopausal women in the ABCSG-16 trial. Cancer Res 2018; 78: GS3-01-GS03-01

[37] Bayer CM, Beckmann MW, Fasching PA. Updates on the role of receptor activator of nuclear factor kappaB/receptor activator of nuclear factor kappaB ligand/osteoprotegerin pathway in breast cancer risk and treatment. Curr Opin Obstet Gynecol 2017; 29: 4-11

[38] Hein A, Bayer CM, Schrauder MG et al. Polymorphisms in the RANK/ RANKL genes and their effect on bone specific prognosis in breast cancer patients. Biomed Res Int 2014; 2014: 842452

[39] Timotheadou E, Kalogeras KT, Koliou GA et al. Evaluation of the prognostic value of RANK, OPG, and RANKL mRNA expression in early breast cancer patients treated with anthracycline-based adjuvant chemotherapy. Transl Oncol 2017; 10: 589-598

[40] Sigl V, Owusu-Boaitey K, Joshi PA et al. RANKL/RANK control Brca1 mutation-driven mammary tumors. Cell Res 2016; 26: 761-774

[41] Kiechl S, Schramek D, Widschwendter $M$ et al. Aberrant regulation of RANKL/OPG in women at high risk of developing breast cancer. Oncotarget 2017; 8: 3811-3825

[42] Nolan E, Vaillant F, Branstetter D et al. RANK ligand as a potential target for breast cancer prevention in BRCA1-mutation carriers. Nat Med 2016; 22: 933-939 
[43] Lee O, Sun L, Karavites L et al. Abstract P5-14-01: Telapristone acetate abrogates PR-dependent paracrine-mediated mammary cell proliferation. Cancer Res 2018; 78: P5-14-01-P15-14-01

[44] Lux MP, Bayer CM, Loehberg CR et al. Shared decision-making in metastatic breast cancer: discrepancy between the expected prolongation of life and treatment efficacy between patients and physicians, and influencing factors. Breast Cancer Res Treat 2013; 139: 429-440

[45] Thiel FC, Schrauder MG, Fasching PA et al. Shared decision-making in breast cancer: discrepancy between the treatment efficacy required by patients and by physicians. Breast Cancer Res Treat 2012; 135: 811-820

[46] Chirgwin JH, Giobbie-Hurder A, Coates AS et al. Treatment adherence and its impact on disease-free survival in the Breast International Group 1-98 trial of tamoxifen and letrozole, alone and in sequence. J Clin Oncol 2016; 34: 2452-2459

[47] Tresp V, Overhage JM, Bundschus M et al. Going digital: a survey on digitalization and large-scale data analytics in healthcare. Proceedings of the IEEE 2016; 104: 2180-2206

[48] Wallwiener M, Matthies L, Simoes E et al. Reliability of an e-PRO Tool of EORTC QLQ-C30 for measurement of health-related quality of life in patients with breast cancer: prospective randomized trial. J Med Internet Res 2017; 19: e322

[49] Dabrock P, Augsburg S. Big Data und Gesundheit - Datensouveränität als informationelle Freiheitsgestaltung (2017). Online: http://www. ethikrat.org/presse/pressekonferenzen/pressekonferenz-30-11-2017; last access: 17.01.2018

[50] Basch EM, Deal AM, Dueck AC et al. Overall survival results of a randomized trial assessing patient-reported outcomes for symptom monitoring during routine cancer treatment. J Clin Oncol 2017; 35 (Suppl.): Abstr. LBA2

[51] Fasching PA, Brucker SY, Fehm TN et al. Biomarkers in patients with metastatic breast cancer and the PRAEGNANT Study Network. Geburtsh Frauenheilk 2015; 75: 41-50

[52] Wallwiener M, HeindI F, Brucker SY et al. Implementation and feasibility of electronic patient-reported outcome (ePRO) data entry in the PRAEGNANT Real-Time Advanced and Metastatic Breast Cancer Registry. Geburtsh Frauenheilk 2017; 77: 870-878

[53] Fasching PA, Wallwiener M, Lux MP et al. Seraphina - Safety efficacy and patient reported outcomes of advanced breast cancer patients: therapy management with NAB-paclitaxel in daily routine. Cancer Research 2016; 76: Abstract OT3-02-09. doi:10.1158/1538-7445.SABCS15-OT302-09

[54] West German Study Group, Commission for translational Research of the AGO. Impact of eHealth-support on quality of life in metastatic breast cancer patients treated with palbociclib and endocrine therapy (PRECYCLE) (2017). Online: https://clinicaltrials.gov/ct2/show/ NCT03220178; last access: 17.01.2018

[55] Overkamp F, Kolberg HC, Schütz F et al. Mammakarzinom Transparent (2017). Online: https://mammakarzinom.onkowissen.de/; last access: 24.01.2018

[56] Muller V, Nabieva N, Haberle L et al. Impact of disease progression on health-related quality of life in patients with metastatic breast cancer in the PRAEGNANT breast cancer registry. Breast 2018; 37: 154-160

[57] Hurvitz SA, Lalla D, Crosby RD et al. Use of the metastatic breast cancer progression (MBC-P) questionnaire to assess the value of progressionfree survival for women with metastatic breast cancer. Breast Cancer Res Treat 2013; 142: 603-609

[58] ClinicalTrials. Study to assess the safety and efficacy of ribociclib (LEE011) in combination with letrozole for the treatment of men and pre/postmenopausal women with HR+ HER2- aBC. Study to assess the safety and efficacy of ribociclib (LEE011) in combination with letrozole for the treatment of men and pre/postmenopausal women with $\mathrm{HR}+$ HER2- aBC (RIBANNA-study) 2018. Online: https://clinicaltrials.gov/ct2/ show/NCT02941926; last access: 17.01.2018
[59] Fasching PA, Ekici AB, Adamietz BR et al. Breast cancer risk - genes, environment and clinics. Geburtsh Frauenheilk 2011; 71: 1056-1066

[60] Fasching PA, Ekici AB, Wachter DL et al. Breast cancer risk - from genetics to molecular understanding of pathogenesis. Geburtsh Frauenheilk 2013; 73: 1228-1235

[61] Couch F], Hart SN, Sharma P et al. Inherited mutations in 17 breast cancer susceptibility genes among a large triple-negative breast cancer cohort unselected for family history of breast cancer. J Clin Oncol 2015; 33: 304-311

[62] Couch F], Shimelis H, Hu C et al. Associations between cancer predisposition testing panel genes and breast cancer. JAMA Oncol 2017; 3: 11901196

[63] Couch F, Shimelis H, LaDuca H et al. Abstract PD1-01: Triple negative breast cancer predisposition genes. Cancer Res 2018; 78: PD1-01PD01-01

[64] Shimelis H, Mesman RLS, Von Nicolai C et al. BRCA2 hypomorphic missense variants confer moderate risks of breast cancer. Cancer Res 2017; 77: 2789-2799

[65] Southey MC, Goldgar DE, Winqvist R et al. PALB2, CHEK2 and ATM rare variants and cancer risk: data from COGS. J Med Genet 2016; 53: 800811

[66] Schmidt MK, Hogervorst F, van Hien R et al. Age- and tumor subtypespecific breast cancer risk estimates for CHEK2*1100delC carriers. J Clin Oncol 2016; 34: 2750-2760

[67] Pelttari LM, Khan S, Vuorela M et al. RAD51B in familial breast cancer PLoS One 2016; 11: e0153788

[68] Fasching P, Hu C, Hart S et al. Abstract PD1-02: Cancer predisposition genes in metastatic breast cancer - association with metastatic pattern, prognosis, patient and tumor characteristics. Cancer Res 2018; 78: PD102-PD01-02

[69] Couch F, Shimelis H, Hart S et al. Abstract GS4-06: Cancer risks and response to targeted therapy associated with BRCA2 variants of uncertain significance. Cancer Res 2018; 78: GS4-06-GS04-06

[70] Milne RL, Kuchenbaecker KB, Michailidou K et al. Identification of ten variants associated with risk of estrogen-receptor-negative breast cancer. Nat Genet 2017; 49: 1767-1778

[71] Michailidou K, Lindstrom S, Dennis J et al. Association analysis identifies 65 new breast cancer risk loci. Nature 2017; 551: 92-94

[72] Ghoussaini M, French JD, Michailidou K et al. Evidence that the 5p12 variant rs10941679 confers susceptibility to estrogen-receptor-positive breast cancer through FGF10 and MRPS30 regulation. Am J Hum Genet 2016; 99: 903-911

[73] Couch F], Kuchenbaecker KB, Michailidou K et al. Identification of four novel susceptibility loci for oestrogen receptor negative breast cancer. Nat Commun 2016; 7: 11375

[74] Purrington KS, Slager S, Eccles D et al. Genome-wide association study identifies 25 known breast cancer susceptibility loci as risk factors for triple-negative breast cancer. Carcinogenesis 2014; 35: 1012-1019

[75] Stevens KN, Fredericksen Z, Vachon CM et al. 19p13.1 is a triple-negative-specific breast cancer susceptibility locus. Cancer Res 2012; 72: 1795-1803

[76] Haberle L, Hein A, Rubner M et al. Predicting triple-negative breast cancer subtype using multiple single nucleotide polymorphisms for breast cancer risk and several variable selection methods. Geburtsh Frauenheilk 2017; 77: 667-678

[77] Mavaddat N, Pharoah PD, Michailidou K et al. Prediction of breast cance risk based on profiling with common genetic variants. J Natl Cancer Inst 2015; 107: pii: djv036. doi:10.1093/jnci/djv036

[78] Vachon CM, Pankratz VS, Scott CG et al. The contributions of breast density and common genetic variation to breast cancer risk. J Natl Cancer Inst 2015; 107: pii: dju397. doi:10.1093/jnci/dju397 
[79] Rudolph A, Song M, Brook MN et al. Joint associations of a polygenic risk score and environmental risk factors for breast cancer in the Breast Cancer Association Consortium. Int J Epidemiol 2018. doi:10.1093/ije/ dyx242

[80] Guo Q, Burgess S, Turman C et al. Body mass index and breast cancer survival: a Mendelian randomization analysis. Int J Epidemiol 2017; 46: 1814-1822
[81] Zhang B, Shu XO, Delahanty RJ et al. Height and breast cancer risk: evidence from prospective studies and Mendelian randomization. J Natl Cancer Inst 2015; 107: pii: djv219. doi:10.1093/jnci/djv219

[82] Vachon CM, Scott CG, Fasching PA et al. Common breast cancer susceptibility variants in LSP1 and RAD51L1 are associated with mammographic density measures that predict breast cancer risk. Cancer Epidemiol Biomarkers Prev 2012; 21: 1156-1166 